\title{
A NETWORK COMBINING PACKET SWITCHING AND TIME DIVISION CIRCUIT SWITCHING IN A COMMON SYSTEM
}

Joe de Smet, Consultant, \& Ray W. Sanders, Computer Transmission Corporation

Digitai data transmission systems have quite rationally followed the patterns established by the older voice and telegraph transmission systems. Thus we find the more complex data transmission systems in use today are generally based on dial-up telephone networks or on polling systems over multipoint channels as used in earlier telegraph and teletypewriter networks.

The need to consider new approaches became apparent in studying the digital communication requirements of the State of California. Like governments in the world today, the use of computers has grown dramatically over the past few years. The need for remote access to and from these computers has grown exponentially. Each agency has been solving its own data communication problems based on voice grade dial or on digital polling schemes or a combination of both. As could be expected, growth of individual overlapping networks resulted in an inefficient maze when viewed as an overall system.

In 1973, the California State Universities and Colleges (CSUC) published a system description based on CSUC's present and forecasted data transmission requirements. This proposed system combined the attributes of voice type dial networks with digital on line call routing of the polling systems. Connections would be established by digital signals on line, but once a connection was established there would be no dependence on the protocol of the transmitted data by the switching media. Additional features stipulated included error correction, 300 millisecond turnaround time, and the integration of asynchronous time share traffic with synchronous batch traffic for line transmission efficiency.

Using this CSUC proposal as a base and reviewing the existing data systems in use by other agencies, The Pacific Telephone Company, one of the Bell system operating companies, in cooperation with the State Department of General Services developed the following features as a basis for a potential statewide interdepartmental data network. The network was designed to be capable of growing to meet the data transmission needs of all agencies.

Let's take a look at the interfaces required for a fully integrated system. From a user terminal point of view, such a system must provide basic supervisory and control functions. That is, it must be able to turn the user terminal on and off. It must recognize demand for service from the terminal. It must establish connections to other terminals or computer ports either on a demand or on a prescription basis. 
Also for maintenance of service it must provide a means of self checking and for remote testing.

Additional terminal features of an integrated system should include: autobaud, echoplex, abbreviated dialing, service queue with automatic cut through for busy connections, as well as service messages for failed lines or down computers.

Any new system should, of course, be capable of supporting all varieties of terminals now in use; but it should also provide flexibility for supporting new and unknown terminals without extensive reprogramming.

At the start, a new system must fully support teletypewriters (TTY) and cathode ray tubes (CRT) in both time share and inquiry response services. It must also simultaneously support remote job entry (RJE) devices in remote batch service. As the former are generally asynchronous while the latter are generally synchronous, both modes must be supported.

Interfaces to computer ports will require essentialiy all of the above user terminal features plus the ability to band groups of ports so they may be addressed with a single acronym. In addition, a multiplex scheme should be available so that several remote terminals can be simultaneously delivered to one computer port for software demultiplexing.

From an overall system point of view, if maximum line efficiency is to be obtained, traffic from various terminals must be consolidated on one line and routed to distant terminats or computers. A network of switching nodes, each capable of concentrating and routing traffic, is an obvious requirement. Such switching nodes must provide the terminal interfaces mentioned above. They must also concentrate, multiplex and switch data on demand or by pre-assignment. Switching nodes must be interconnected by data links or lines. Nodes must switch data traffic either directly or via other nodes for efficient traffic handling and minimum line costs. Interface to such data links should include error detection. and correction, synchronous and asynchronous terminals served over the same data link, and automatic alternate routing for traffic overload or line failure.

In order to make a viable system, we must specify certain limits for call connection and data delivery. These include partitioning, priority, set-up time, and response time.

An integrated system must provide a means of preventing illicit connections as well as for establishing desirable connections. This ability to restrict access is called partitioning. Both flexibility in assignment and security in operation are fundamental 
requirements for partitioning. The security must be of a nature that even if the modus operandi becomes known, security will still be maintained.

Priority is required between various groups or agencies. For example, law enforcement may need a completely unblocking system while college student time share service might afford ten second, or more likely five minute, delay in call set-up. Priority should also be available within individual groups. For example, administrative messages might take precedence over routine traffic between the same subset of stations. Dynamic priority of data link bandwidth must also be available. Time share and inquiry response service demand immediate attention while batch traffic can be delayed.

Set-up time was not found to be critica 1; however, the traffic offered indicated that ten connections per second must be established by the system. Response time was defined as the interval after the last bit of a character from a terminal is received at a switch node until the start of the first bit of that character back toward the same terminal with the distant switch looped back at a terminal interface. This round trip delay was requested by CSUC as not more than 300 milliseconds.

Even while the new system was being defined, additional customer requirements became evident. This indicated a need for extreme modularity and flexibility for growth in new and unsuspected directions. It also became apparent that a communication system which had to follow and react to the variety of line protocols now in use by the computer industry was doomed to everlasting reprograming. Following a review of these factors and the variety of requirements discussed above, a systems philosophy was evolved:

A11 interfaces would meet the EIA RS 232C/CCITT V.24 standard.

Only ASCII and EBCDIC codes would be specified as requirements.

No speed or code conversion would be provided.

The new system would be transparent. That is, once a connection has been established, the data transmitted would be delivered to the receiving station exactly as transmitted by the sending station. Any and all line protocols would be up to the two connected stations.

All connections through the system would be completely ful1 duplex. Whether or not stations sent simultaneously in both directions would be entirely up to the connected stations.

The new system must be completely modular. It must be capable of being changed without disruption of existing data services. Not only must it be modular so that stations could be added or deleted, but so that data link and data switches could be added, deleted or rearranged while other stations and switches are enjoying service without interference. 
Last, but by no means least, the system must be maintainable. This means, maintainable at remote locations where maintenance men skilled in complex data systems are not readily available. A network control center capable of down line assignment changes, network self test features with centralized reporting, and complete down line test capability were deemed as mandatory. The network itself must be self checking and self healing to the extent feasible, and above all must report any deviations to the network control center.

Following development of the above features and philosophy, a search was initiated for existing hardware and software which could be adapted to provide a statewide integrated data system. While not dwelling on the selection process, it might be well to note that relatively standard steps were followed in issuing a request for price quotation ( $R P Q)$, evaluating the proposals received, and on the second attempt finding a system which could economically meet these specifications. This is the Computer Transmission Corporation TRAN (B) M3200 system.

The M3200 Network Switching and Management System PACUIT atm switching has been developed to provide the advantages of both circuit-switching and packet-switching, while eliminating many of their disadvantages. The fundamental design objective is that of minimizing delays in the network. To this end, the M3200 system incorporates many important new concepts, in conjunction with the best established techniques from TRAN's extensive experience in implementing networks. Highlights of the system are summarized below.

a. The PACUIT switching concept for reducing node buffering requirements by providing cyclic redundancy checking only at source and destination nodes.

b. Synchronous master-clock operation on 211 internode trunks.

c. Concurrent transmission of PACUITized data and simple time division circuitswitched data (non-PACUITized), on the same trunk.

d. Dynamic bandwidth allocation for optimum utilization of internode trunks, based on statistical multiplexing.

e. Optional fixed bandwidth for dedicated channels.

f. "Dynamic multiplexing option" available on tail-circuits (i.e., local distribution from a node). for efficient line utilization.

g. Fu11 duplex transmission implemented by independent (simplex) transmission of data messages/responses in each direction.

TRAN $Q$ is a registered trademark of Computer Transmission Corporation

PACUIT $\mathrm{tm}$ is a trademark of Computer Transmission Corporation 
h. Highly efficient PACUIT acknowledgement technique, for minimizing delays when transmission retries are necessary.

i. Alternate routing capability when individual trunks are overloaded, or giving high error rates, or are out of service.

j. Continuous performance monitoring of traffic and error rates.

k. Port selection and contention for synchronous and/or asynchronous traffic, to either individua? ports or "hunt groups".

1. Logical network partitioning into user "access groups" for security.

m. "Abbreviated diatmin" within access group, for fast resource selection from a terminal.

n. "Camp-on" queuing of new users (if necessary) to avoid user retries during peak traffic periods.

o. Network statistics and diagnostics available on system console at each node.

p. Preassigned network management bandwidth for efficient system control.

q. Network Management System available (intended for large networks) for complete centralized control, including statistics, diagnostics, and network reconfiguration.

$r$. Basic network is transparent to user protocol and code to avoid overloading switches. Protocol and/or code conversion can be added to switch modules or performed in separate utility modules, connected to one or more nodes as necessary.

s. Switching function and PACUIT assembiy/disassembly are assisted by specially designed microprocessors, for optimum node efficiency.

t. General purpose bus architecture of switch allows highly flexible configuration capability, giving significant economic advantages and easy expandability.

\section{PACUIT - THE HYBRID APPROACH}

A major advantage of packet-switching is that of powerful error checking and recovery. However, the classical means of forming and transmitting packets is a significant source of transmission delay and excessive network overhead for low speed terminal traffic. Circuit-switching systems do not provide error checking, and therefore do not suffer from such delays and network inefficiencies. To provide the best of both systems, TRAN has introduced the hybrid PACUIT technique:

$$
\text { PACket }+ \text { circUIT }=\text { PACUII }
$$

A PACUIT is a highly efficient packet structure containing data traveling between the same source and destination nodes. The PACUIT structure is a variable length entity which effectively compacts data in an optimum manner. Because all data in a PACUIT have common source and destination nodes, there is no need to perform error checking or to store packets at each intermediate node along the path. Instead, only the source 
and destination switches perform packet control functions, while all intermediate nodes operate in a purely time division circuit-switched mode for through traffic. That is, acknowledgement is performed only on a network end-to-end basis. The effect of this is to minimize end-to-end delays, while reducing the buffer storage requirements at each intermediate node. The latter follows from queuing theory in that the less time PACUIT's spend in each node, the less that queues will build up. The logical link established between any source/destination node pair is referred to as a "virtual connection".

Buffer storage requirements at end nodes are also reduced, as a result of another difference between the PACUIT and packet approaches. This is that PACUIT's are transmitted at regular intervals on each virtual connection emanating from the source node, whereas packets (in most earlier systems) are not transmitted until they contain a certain minimum amount of data. To understand why this is important, it must be remembered that data enters or leaves end nodes (to/from resources) at various rates.

In pure packet systems, the time that each buffer remains allocated depends on this rate. Since buffer sizes are predefined (e.g., equal to the minimum packet size), slow terminals may tie up considerable amounts of buffer storage for relatively long periods.

In the M3200 system, however, only smal1 buffers need be allocated, since they will be emptied at regular intervals (each time a PACUIT is formed and transmitted). If no data is entered from a particular terminal between transmission of one PACUIT and the next, almost no space will be wasted for it in the new PACUIT. In other words, the PACUIT compaction technique ensures a minimal overhead for inactive terminals. It is also worth noting here that when a terminal completely disconnects from the network, it is completely de-allocated from the PACUIT channel.

Since PACUIT's may contain multiple-channel data, a well chosen transmission rate will result in a high probability that some channels witl have data to send, even if others have not. Referring to Figure 1, the mean data content of a PACUIT must clearly be sufficiently high to ensure that the percentage overhead (due to structural information) is low. On the other hand, it must not be too high; otherwise the advantage of low buffer requirements will be lost.

The PACUIT transmission, rate is, in fact, dynamically controlled by the M3200 switch software, using a monitoring and feedback technique. The same technique is used to control dynamic bandwidth allocation, and is explained in more detail below.

As was previously indicated, the use of packetizing is not always necessary. For transmission protocols which include their own powerful error detection codes, the use of packets is not as easily justified as it is for nonchecking protocols. In practice, it 


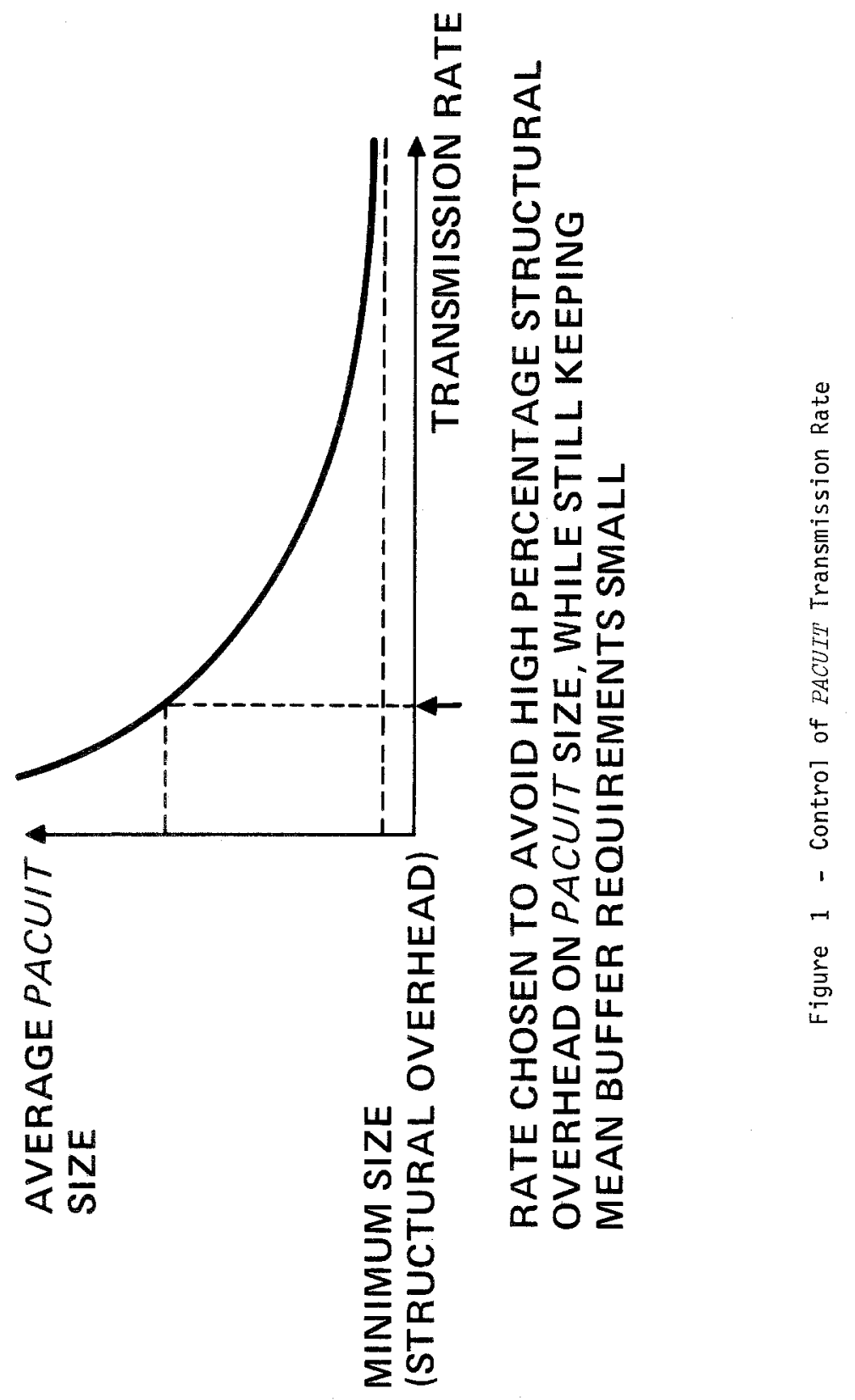


is generally useful to packetize asynchronous data, since asynchronous terminals tend to offer weak or no error checking. Synchronous terminals, however, often include powerful detection codes such as cyclic redundancy checking.

The M3200 is the first system to offer the ability for mixing both packetized (PACUIT) data and individual time division circuit-switched channels, concurrently on the same internode trunks (see Figure 2).

STRUCTURE OF NODAL EQUIPMENT

The Nodal Equipment consists of two major units - a Digital Switch and a stored Program Controller.

The Digital Switch provides all data 1/0 to both terminals and lines while the Controller controls all assignments in the Digital Switch.

For example, a terminal requesting service would be automatically connected through the Switch to the Controller. The controller would determine routing and notify the Digital Switch which output to connect to the terminal. Al1 data originated by that terminal will continue to be relayed in both directions by the Digital Switch without further reference to the controller, regardless of the data content. Disconnect is handled in a similar manner.

The Controller obtains all routing information from programmable tables. It also provides continuous test in background and logs traffic information.

\section{DYNAMIC BANDWIDTH ALLOCATION}

The technique used for intermixing PACUIT and pure circuit-switched data on trunk lines is that of time division multiplexing on a character-by-character basis. In earlier systems, the TDM technique results in less efficient trunk bandwidth utilization. This is due to the fact that time slot allocations are fixed within the repetitive channel selection sequence used for inserting data on the line. Thus, while no data is being entered from a terminal, its time slots will be unused, and are generally filled by "idle" characters.

TRAN has developed a new technique for the M3200 system which permits the dynamic reassignment of time slots to different data sources. This technique is knows as "dynamic bandwidth allocation". The decision to perform reassignment is based on a data traffic monitoring method referred to as "statistical multiplexing". This is implemented in the M3200 system by software in the switch minicomputer. The statistical multiplexing routines within a. source node monitor the outgoing traffic at frequent intervals, on a channel-by-channe1 basis. In this case, each active PACUIT connection is treated as a single channe1. The technique is, in effect, a "feedback" system - the higher a 


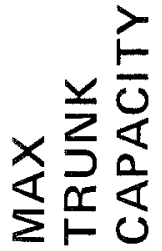

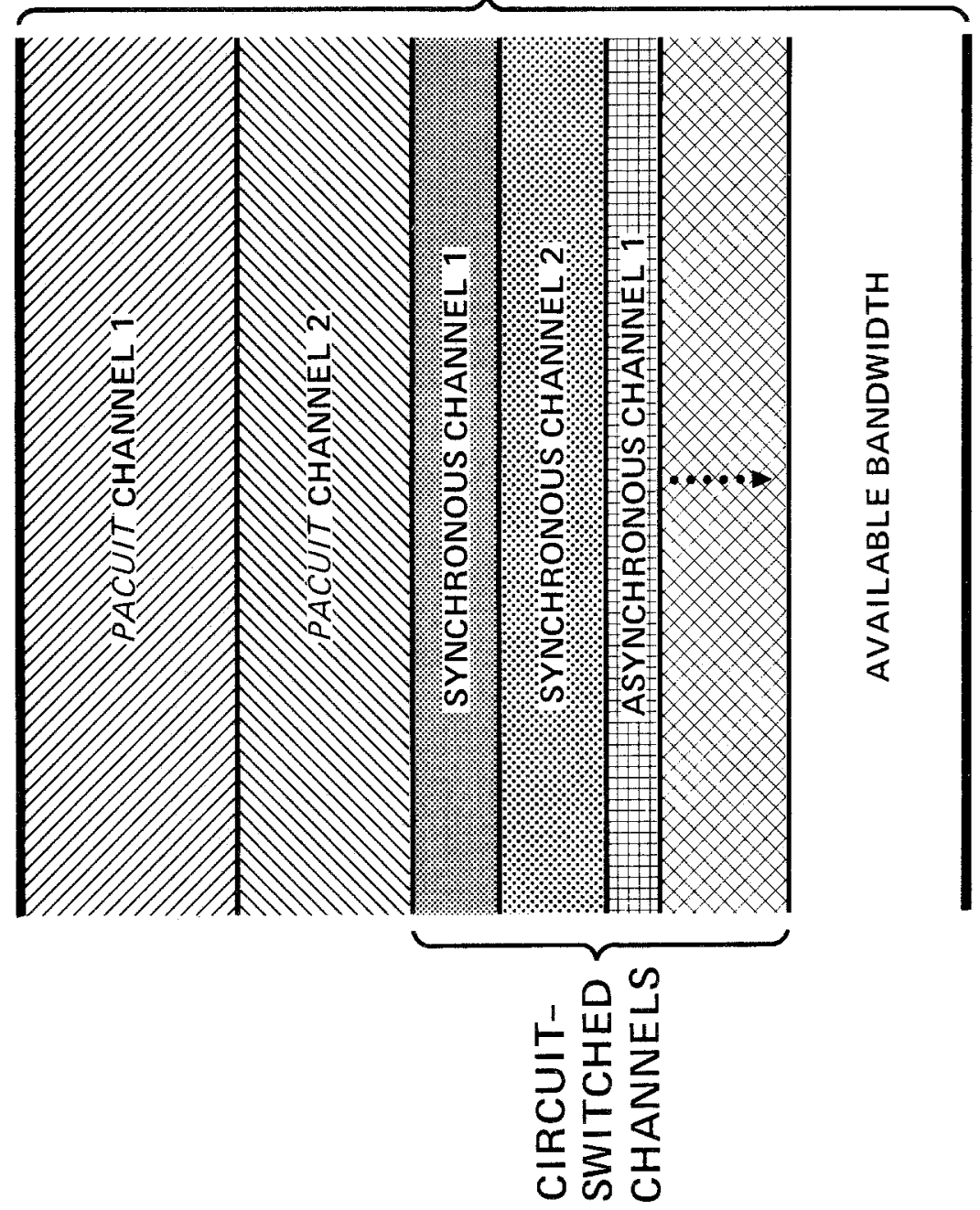

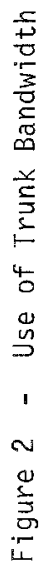


channel composite traffic rate, the greater the bandwidth allocated to it; and viceversa.

The M3200 system offers two types of synchronous circuits, one with flow control, and one without flow control (fixed bandwidth). For flow controlled synchronous channels, message transmission results in bursts of data from a buffer. In this case though, the buffer is that of the originating terminal, and not of its network entry node. The rate at which a synchronous terminal buffer is emptied is dependent on the available transmission capacity. A Remote Terminal Interface Unit (RTIU) controls the terminal clock to make use of this capacity. An RTIU may be used between each synchronous terminal and its point of entry to the network if circuit-switched service is required. The unit also serves a second function, that of resource addressing. If fixed bandwidth, dedicated (i.e., non-switched) service is required, then no RTIU is necessary.

\section{DYNAMIC MULTIPLEXING}

When several terminals are grouped together at some distance from their nearest node of an $\mathrm{M} 3200$ network, they may enter the network via a multiplexed tail-circuit (see Figure 3). In order to make optimum use of the tai7-circuit trunk bandwidth, a technique is used that resembles dynamic bandwidth allocation in that it allows continual reassignment of time slot positions in the multiplexed channel selection sequence. The technique is called "dynamic multiplexing". A current specification of time slot/ channel assignments is reloaded dynamically from the M3200 switch.

The effect of dynamic multiplexing is that the tail-circuit trunk bandwidth may be considerably less than the sum of the bandwidths of all terminals attached to the multiplexers. This can result in considerable cost savings, where line and modem costs are bandwidth-dependent.

\section{FULL DUPLEX OPERATION}

Trunk 1 ines between M3200 nodes are both physically and logically full duplex. That is, they are capable of carrying data or response information in both directions concurrentiy. Moreover, data and responses need not be alternating. In the case of a synchronous circuit-switched channel, this will depend on the originating terminal type. For example, most Binary Synchronous Communication (BSC) terminals do not, in fact, work in a true ful1 duplex manner. At best they perform in a "conversational" manner, with alternating messages in each direction (i.e.., an output message may be sent instead of a positive acknowledgement to the preceding input message).

PACUIT operation, however, is logically full duplex. Consecutive PACUIT's may be transmitted in either direction, without waiting for the response to each one in turn. A proprietary TRAN technique permits a single five-bit response to fully identify the sequence of acknowledgements (positive and/or negative) up to four consecutive received 


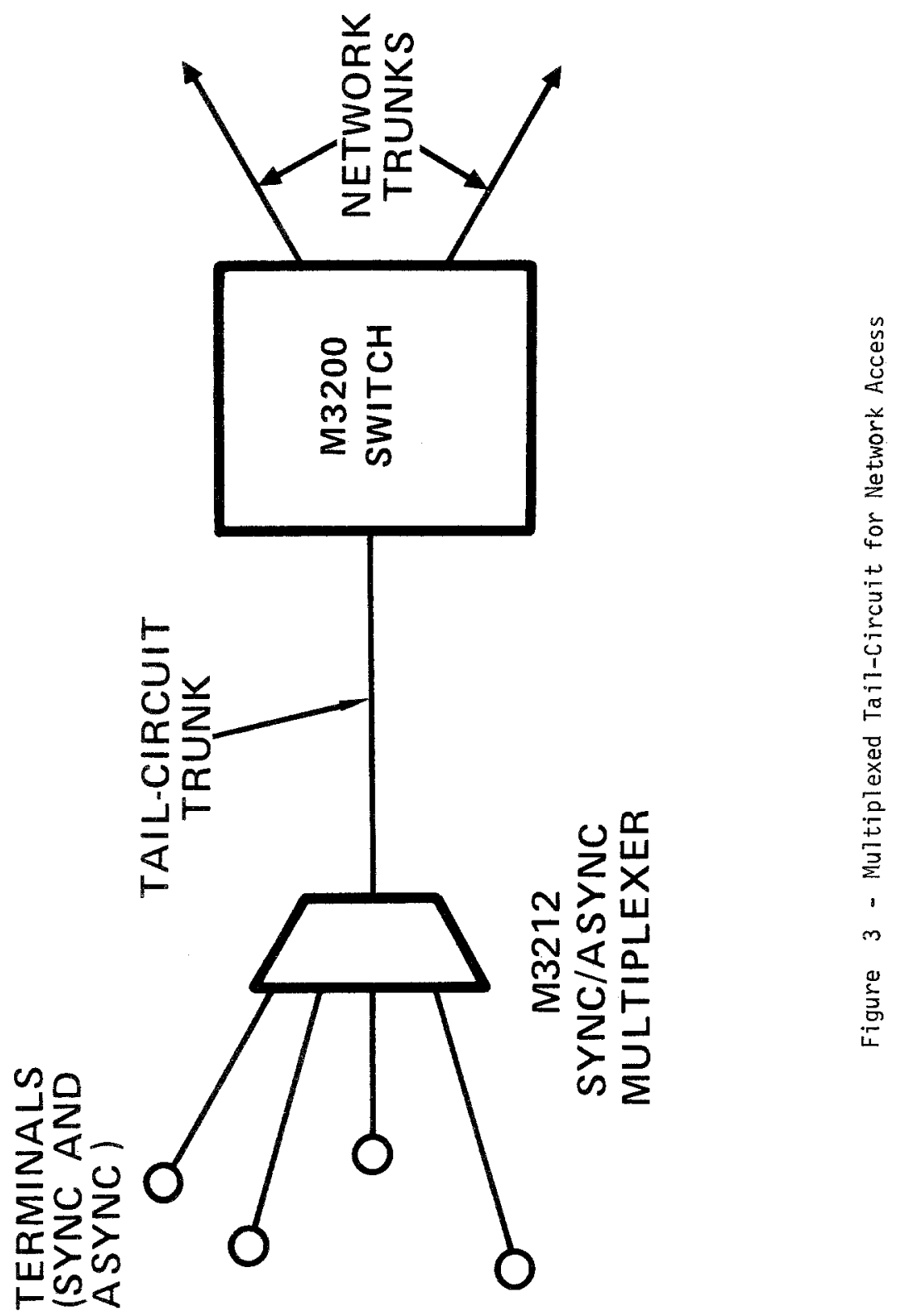


PACUIT's. Such a response is usually carried within a PACUIT being transmitted on the same connection, in the opposite direction to the received PACUIT's. This is illustrated in Figure 4 . Labels A1, B1, etc., represent PACUIT numbers, while RAn, RBn, etc., represent the single or accumulated responses. Note in this example that response RA2-4 identified good receipt of PACUIT's A2 and A3, but an error in A4. The latter is therefore retransmitted.

Such is the independence of the two directions of a PACUIT virtual connection, that they may not even follow the same physical trunk paths. In other words, the fuil duplex capability is implemented by independent simplex transmission of data in each direction. Thus, in Figure 5, PACUIT's traveling from node $A$ to node $D$ pass along the path ABD, while PACUIT's traveling from D to A use the path DCA. Simplex operation is particularly advantageous for the new class of split-speed terminals which operate up to 300 bps on the transmit side, and up to 9600 bps on the receive side.

\section{ALTERNATE ROUTING}

The term "virtual connection" is used to describe the logical link between any node pair for an active user channel. Thus, at the time network operation is started from cold, no virtual connections exist. Normally, the first ones to be established will be those for preassigned dedicated channels. Subsequently, as each new circuitswitched or PACUIT channel is initiated, it will either be added to an existing physical connection, or (if necessary) a new physical connection will be established to "contain" it.

Along the path of an established physical connection, the source and destination nodes are logically defined to the switch software as being PACUIT assembly/disassembly nodes. Any intermediate nodes are set up for time division circuit-switching only. (Note that an intermediate node of one physical connection may also be the source node of another.)

A source node is responsible for control of the connection. In the first place, the physical connection is set up by the source node minicomputer, based on optimum path tables contained in the software. The choice of the physical route depends not only on geographical considerations, but also on the current status of the relevant internode trunks. If any trunk in the potential route is either too heavily loaded, or out of service, an alternative route will be selected.

The connection approach used guarantees that no circuit-switched channels will be "dropped", in the event of trunk failures during operation. Any such failure (including detection by any node along the path of an unacceptable error rate) will be signaled to the source node, which will then set up an alternative route, and switch traffic onto it. 

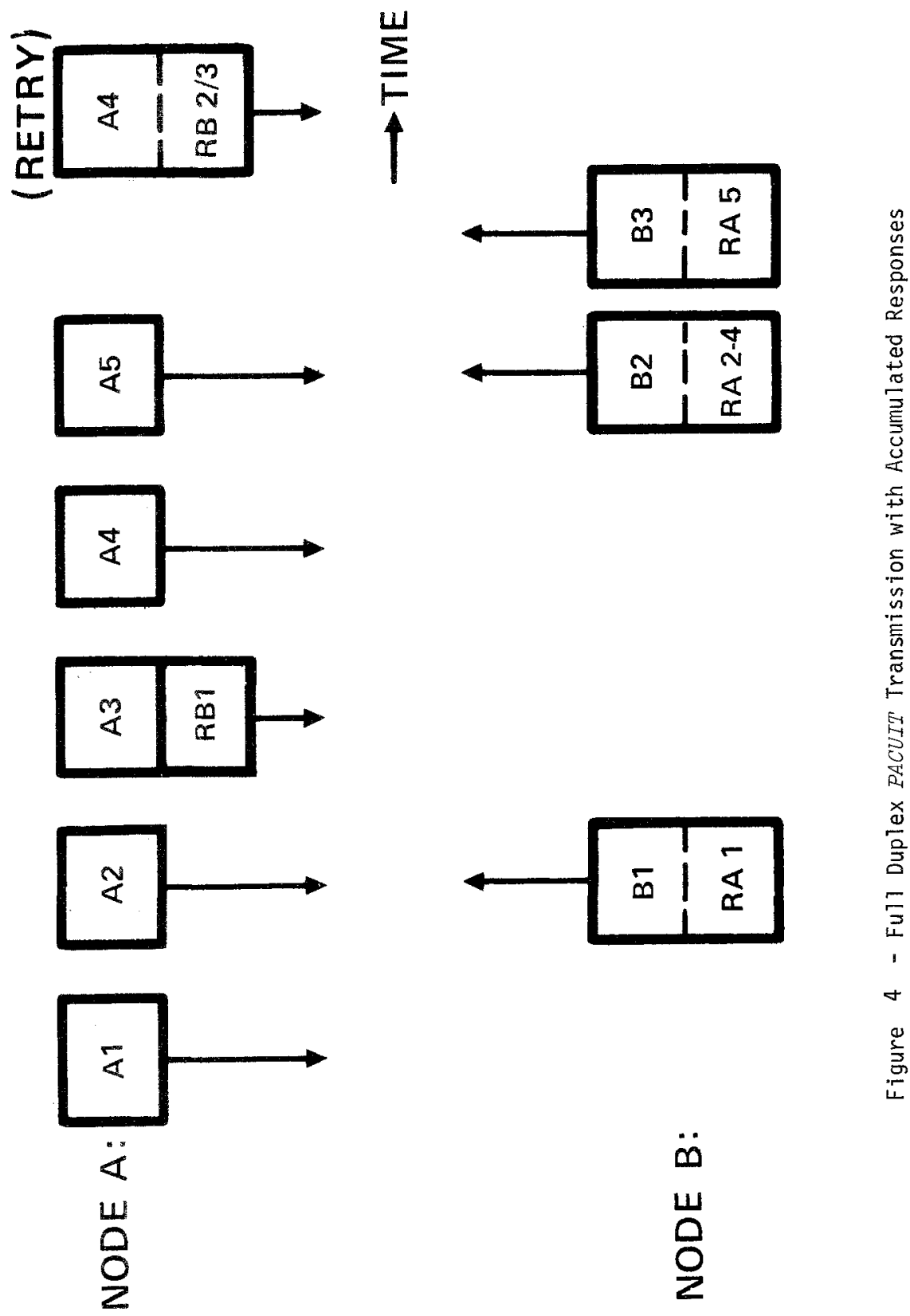

$\ddot{\oplus}$

을 


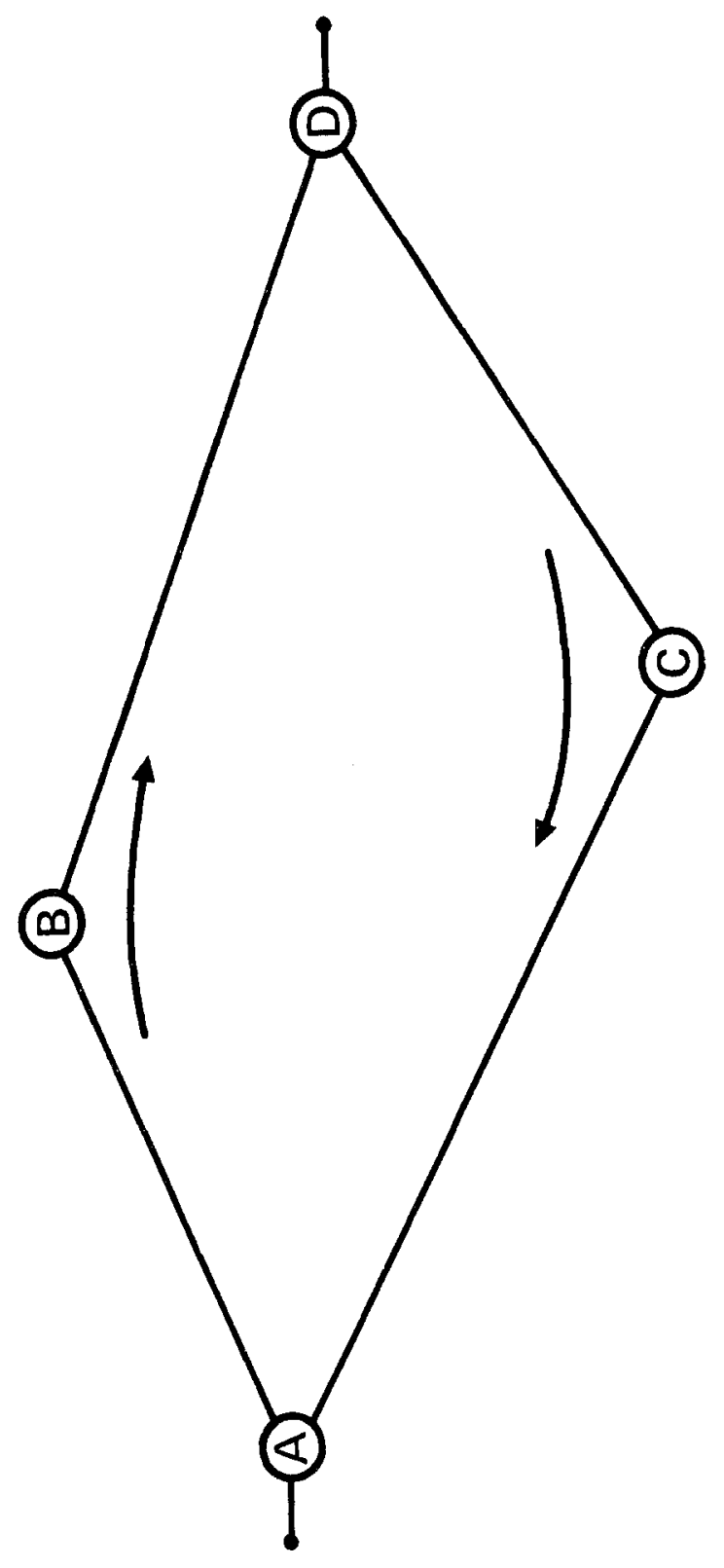

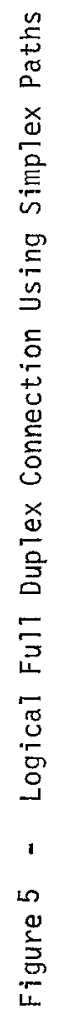




\section{NETWORK ACCESS AND PARTITIONING}

It is worth recalling, at this point, that the fundamental objective of a switched network is to allow one resource to select and access another resource le.g., terminal to computer), in a simple secure manner. The $M 3200$ system provides a number of features designed with this objective in mind. These features may be classified according to the general functions to which they contribute.

a. Access Security

1. "Access group" specification

2. Logical network partitioning

b. Resource Identification

1. Abbreviated dial-in

2. Selection by specific identifier or "hunt group"

3. Symbolic identifiers

4. Remote Terminal Interface Unit (RTIU) with "touchpad" keys (for synchronous terminals)

c. Connection Assistance

1. "Autobaud" line-speed recognition

2. Preassigned bandwidth characteristics (common, dedicated, or reserved)

3. Other preassigned characteristics such as "priority access", "emergency access", etc.

4. "Camp-on" queuing for unavailable resources

5. Resource messages

d. Additional Services

1. Broadcast messages

2. Message "echo"

3. Local loop parity checking

\section{a. Access Security}

A11 resources connected to an $M 3200$ network are assigned a logical station number by which they may be called, and a set of attributes which govern the resulting connection. Sets of station numbers may be defined as logical "access groups" to the switch software. In most cases, any resource may only connect to a member of its owr access group.

However, selected resources may have capability for calling destinations in other specific groups. The limitation is that the requested station is defined as being able to receive the external cali.

The access group facility effectively provides a means of partitioning the physical network into a set of smaller logical networks. Each of these logical networks may be entirely independent, or may have limited access to others. Partitioning is an 
important facility for networks that are shared by several independent organizations for exampie, public data network services offered by the telephone companies.

\section{b. Resource Identification}

In a partitioned network, the number of resources in an access group is usually a small proportion of the total system resources. Thus, the number of characters or digits required to specify intragroup addresses may be somewhat less than the number required for intergroup addressing. This is analogous to the public telephone network in which seven digits are usually required for dialing a local call; whereas ten are required for long distance calls. The analogy is, however, incomplete inasmuch as the "abbreviated dial-in" facility of an M3200 network is not dependent on distance.

A further analogy exists between M3200 resource identification and telephone dialing. This is the "hunt group" concept. Often, in the major centers of large companies, a number of telephone trunks will be assigned to the central switchboard. An external caller dialing the company number will not necessarily be serviced on one particular Tine; if the first trunk is busy, then an automatic "hunt" for an unoccupied line will commence. Only if all lines are occupied will the caller receive a "busy" signal.

Analogously, multiple ports in an $M 3200$ network which are in the same access group, and which offer access to similar resources, may be specified as members of a common hunt group. Typicaliy, a hunt group will comprise several ports connected to the same computer, and offering access to the same application program(s). Again, however, the analogy with the telephone service is incomplete, since members of an M3200 hunt group may be widely distributed. For example, in Figure 6 identical time sharing services (TSO) are offered by two IBM computers at different nodes of a network. Nonetheless, the total of fifty ports shown may be specified as members of a common hunt group. A terminal user at node $A$ who simply requests a TSO service (see below), may be connected by the system to either of the computers - he does not know or care which one.

The manner in which a terminal user requests a connection depends on his terminal type. Typically, asynchronous terminals are equipped with keyboards through which resource identifiers may be entered. An identifier can be either the station or hunt group number of the resource; or it may be a symbolic equivalent (i.e., alphabetic instead of numeric). Referring again to Figure 6 , the name TSO would be a useful symbolic identifier. The switch minicomputer software contains tables of all such identifiers and their equivalent group of station numbers. The identifiers are defined as required, for any given network.

Synchronous terminals tend to have more diverse configuration characteristics than asynchronous terminals. For example, an RJE batch terminal does not require a key- 


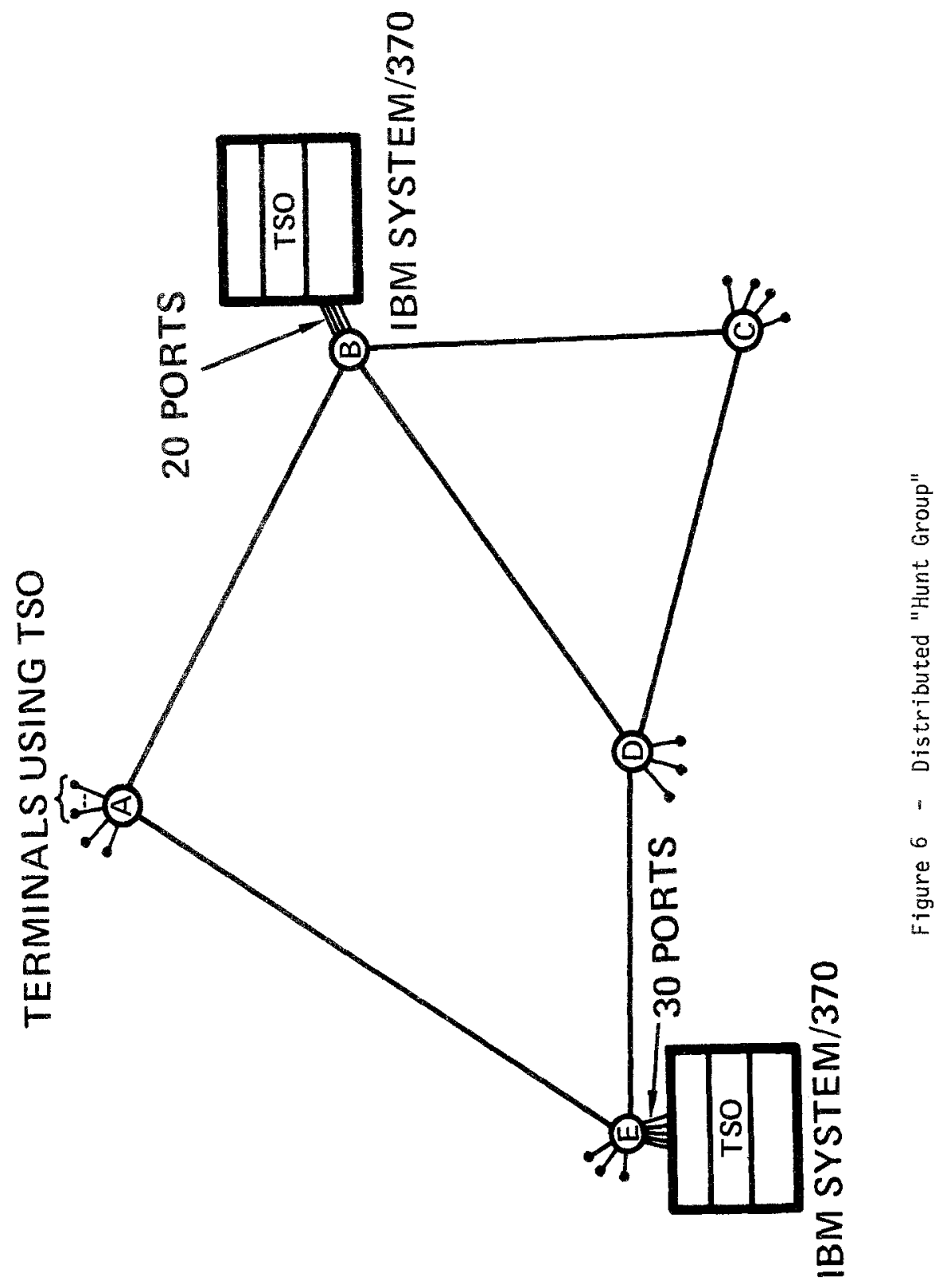


board. For this reason, resource addressing from synchronous terminals makes use of the Remote Terminal Interface Unit referred to before. The RTIU has a "touchpad" keyboard through which resource identifiers may be entered.

\section{SOFTWARE ARCHITECTURE}

The M3200 system runs under a highly efficient real time operating system developed especially for it. This is the Data Switch Operating System (DSOS). The software system, like its hardware counterpart, is modular in structure. Through a system generation process, the functional and $1 / 0$ facilities required by each switch in the network are defined. This allows the building of a tailored version of DSOS for each switch which will be economic in terms of memory requirements, and will have a minimum performance overhead.

The diverse functions to be performed by DSOS are each handled by one or more "tasks". These tasks are grouped into multiple priority "levels", which are almost completely independent of one another. Each task runs under control of a Task Control Block (TCB). The TCB's contain all necessary status information for their related tasks to be run. Because leve1s are independent, each has $i$ ts own Task Dispatcher and queue of readytasks (i.e., pointers to status information for tasks awaiting scheduling). Figure 7 il)ustrates the way in which level and task dispatching operates.

a. Suppose that a hardware interrupt occurs while a task in Level " $n$ " is active. The switch minicomputer uses an automatic vector system for branching to the appropriate entry point in the Interrupt Processor. At the same time, it automatically stores interrupt status information in a queue in reserved memory.

b. The Interrupt Processor immediately calls a "Save" subroutine to locate the Level Control Table (LCT) of the interrupted Level " $n$ " and saves all its volatile infarmation therein (e.g., registers, program counters, etc.).

c. Interrupts are of two basic types:

1. Nonsignificant, which do not require any special servicing (e.g., arrival of a data character from a terminal).

2. Significant, which do require special service (e.g., completion of a message or PACUIT transmission).

In the former case, the Interrupt Processor simply calls "Restore" (to perform the reverse of the "Save" function) and makes an immediate return to the interrupted task in Level " $n$ ". However, for significant interrupts, control is passed to the "Dispatch" routine.

d. "Dispatch" simply performs the minimum necessary housekeeping functions and passes control to Level 1, the Software System Scheduler. The latter is in effect a second-level interrupt handler. It scans all the accumulated interrupt status 


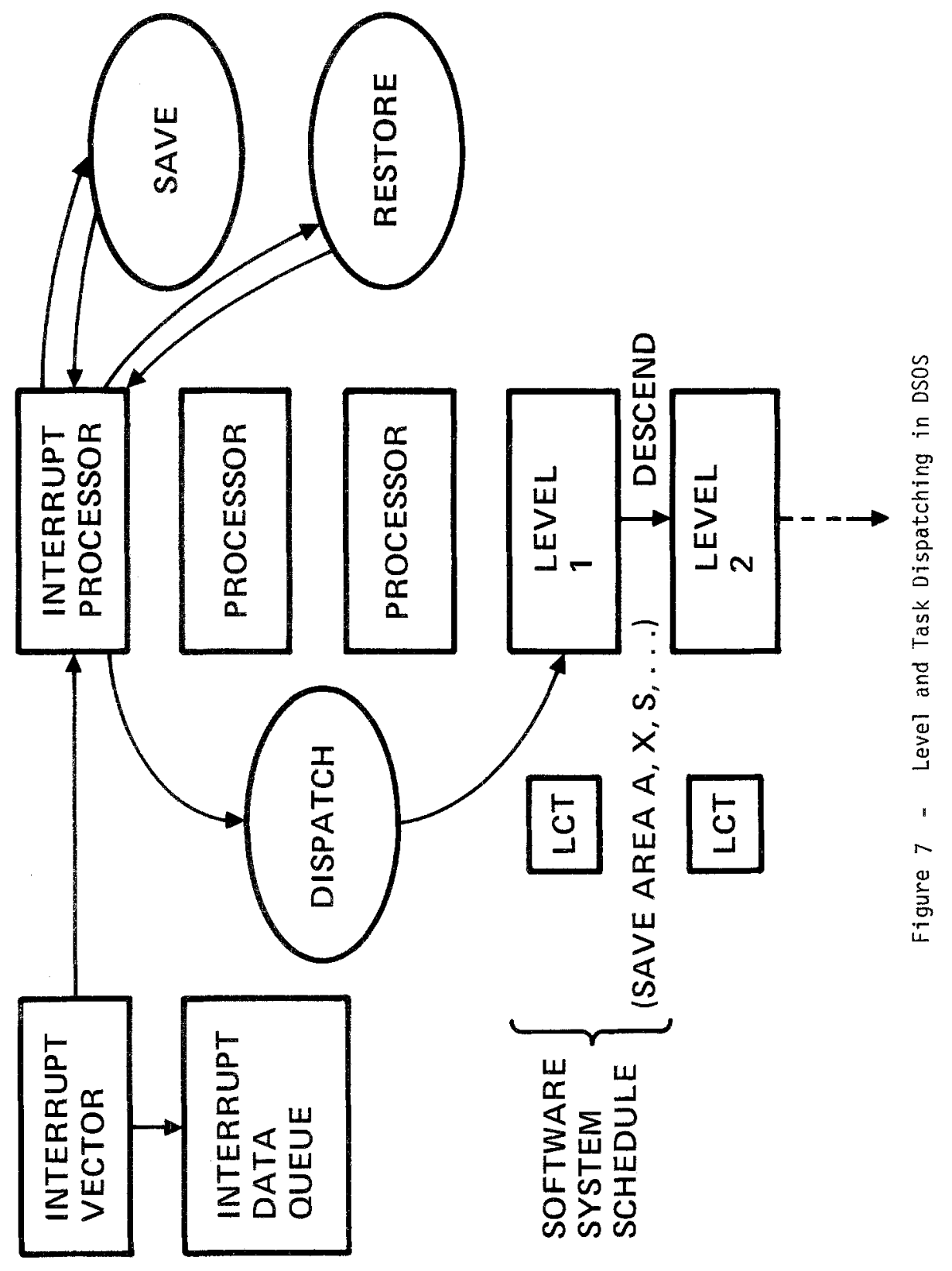


information in reserved memory, and moves it to the appropriate level for later scheduling. This is the function of building the queue of ready-tasks within each level, referred to earlier. Note that the hardware stores status information even when interrupts are lost; hence the need for Level 1 to perform a scan for multiple status elements.

e. After completing its work, Level 1 executes the "Descend" routine. This passes control to the next lower level (2), after restoring volatile information from that lever's LCT.

f. If Level 1 was active when the interrupt occurred (i.e., $n=2$ ), its interrupted task will directly receive control and resume execution. If not, the Task Dispatcher for Level 2 will receive control and will scan its own ready-task queue. If an entry is present, the Task Dispatcher will locate the appropriate TCB (or build one, if necessary) and run the task. As long as no further significant interrupt occurs, all ready-tasks at Level 2 will be run in turn. This done, the Task Dispatcher will execute a "Descend" to the next level (3), and so on.

g. When all tasks at higher levels have been run, the lowest level is entered to perform general network monitoring and diagnostic functions. These tasks always have work to do, and will retain control until a significant interrupt occurs.

The DSOS dispatching mechanism thus ensures that high priority tasks receive immediate service, and that all tasks of any one priority receive service before all lower priority tasks. The current system priority levels are as follows:

a. Software System Scheduler: Distributes status information for level scheduling.

b. Communication Line Level: One TCB for each PACUIT channe7.

c. Error Recovery Leve1: TCB's are generated as required to service error conditions.

d. Connect/Disconnect Leve1: A TCB is generated each time a terminal or port requests a connection or disconnection.

e. Network Management Level: Includes all tasks communicating with the central Network Management System (NMS). If no Network Control Center exists, this level is absent.

f. Operations Level: Tasks include operator communications and all operatorinitiated functions.

g. Background Leve1: Includes initialization, monitoring, and diagnostic tasks.

Many of the actual program functions to be performed by the DSOS software are common to several tasks in a single level, or even to tasks in different levels. To avoid duplication of coding, such functions are programmed as separate "processors" from 
the main task coding. The twelve processors are iliustrated in Figure 8 , which also shows the overall software structure. The nesting structure shown signifies that a high level processor may call one at a lower level, but not vice-versa. Not all calls are shown, since some processors are used by almost all tasks (e.g., the General Table Processor).

The coding of DSOS is fully reenterable, even though the dispatching mechanism only requires serial reusability. This is because the system is essentially table-driven. The major groups of tables are shown at the right of Figure 8 . The software interface to the M3200 hardware architecture is via the two powerful microprocessors shown at the top of Figure 8.

The microprocessors are the PACUIT Processing Unit (PPU) and the Switch Processing Unit (SPU). The PPU functions are structuring and destructuring PACUIT's, at regular intervals for each PACUIT channel. Transmission and reception of both PACUIT and circuit-switched data is handled by the SPU. The latter obtains routing information from Channel Address Tables (CAT's) built and maintained by the software.

As mentioned earlier, many DSOS functions are optional, to allow tailoring of the system to a particular user's requirements. These functions are available in the form of more than thirty optional packages. Adding an optional package to the basic system, in effect, means adding tasks to one or more of the priority levels. Many of the options are related to network diagnostics and management, and are discussed in the next paragraph.

\section{NETWORK TESTING AND DIAGNOSTICS}

Being an "intelligent" system, the M3200 network has many built-in self diagnostic and monitoring capabilities. This "real time" surveillance is automatically initiated by the DSOS software. Active diagnostics, such as loopback tests of available ports, are performed on a time-available basis (in the background level), while monitoring and response to system generated alarms are performed at a high priority level.

Each switch monitors its own internal operation, and the operations of any multiplexers and trunks that it serves. The real time diagnostics are designed to locate most fault conditions before the user is aware of them. The test repertoire includes:

a. Switch diagnostics, with powerful and diverse self tests of the PPU, SPU, minicomputer, and RTD Bus.

b. Trunk monitoring of PACUIT channel error rates to ensure that a user-specified threshold is not exceeded. Also, monitoring for loss of frame synchronization.

c. Port diagnostics, with self testing of channel $1 / 0$ modules and driver/receiver logic. Also, external monitoring such as parity checking on tail-circuits 


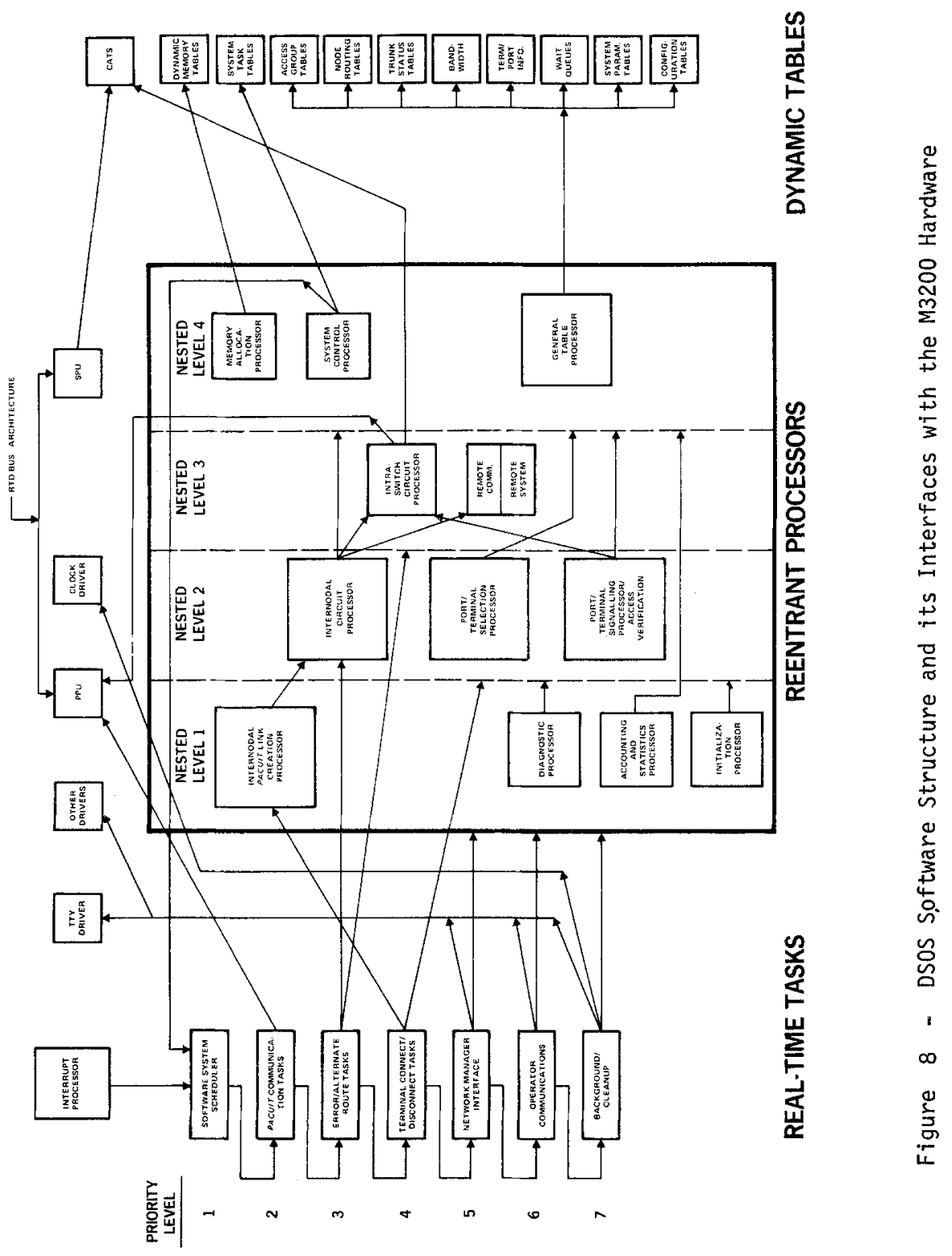


(optiona1), repetitive testing of computer ports that fail to respond to "ringing" by the switch, and remote loopback tests.

d. Configuration checks, to detect insertion of modules in wrong chassis positions or a working system. No module or system damage will be incurred from this error.

When these diagnostic results reveal errors, they may be output at user-specified locations. Typically, in small networks, they will be printed on the teletype console of the switch that diagnosed the error. In larger networks, error alarms will be sent to a Network Control Center.

The local console of any switch node may also be used to initiate certain tests. Many of these operator-initiated tests are similar to those listed above, but are executed with higher priority for the specified resources. In addition, the operator may interrogate the network for channel status information; this test is a connection trace that identifies the destination (if any) of any specified channel. These powerful monitoring and diagnostic capabilities ensure a network reliability and system availability that cannot be matched by traditional online systems.

\section{CONCLUSION}

The results of applying this new TRAN system to the State of California requirements may be summarized:

Many agencies can share one line, thus saving the cost of separate lines and data sets.

Additional savings by integration of synchronous and asynchronous traffic. Efficiency over polling systems, no time lost for data set turn around. Saving in computer ports by providing economy of scale; any port can be accessed from any station so assigned.

Can interface existing polling systems with little or no change. Can provide control for future systems thus eliminating expensive controllers at each station.

Improved service due to error detection and retransmission, queuing, improved access.

Getting started costs for the initial few hundred stations comparable with the cost of existing systems. Incremental costs decrease as the systems grows.

This system has now been tested. The initial service starts March 1, 1976, for the California State Universities and Colleges. Four switches and nineteen multiplexers will serve one hundred fifty stations remotely to two computers for time share and remote batch service. Within two months thereafter, a second agency with additional 
computers and sixty stations will be added to the net. Figure 9 is a sketch of the initial layout. Figure 10 shows the expected three-year growth.

Here then is a digital system which improves service and is competitive in cost for a small layout. It has the desirable feature of decreased per station costs as the network grows combined with the flexibility to meet virtually any data transmission requirement. 


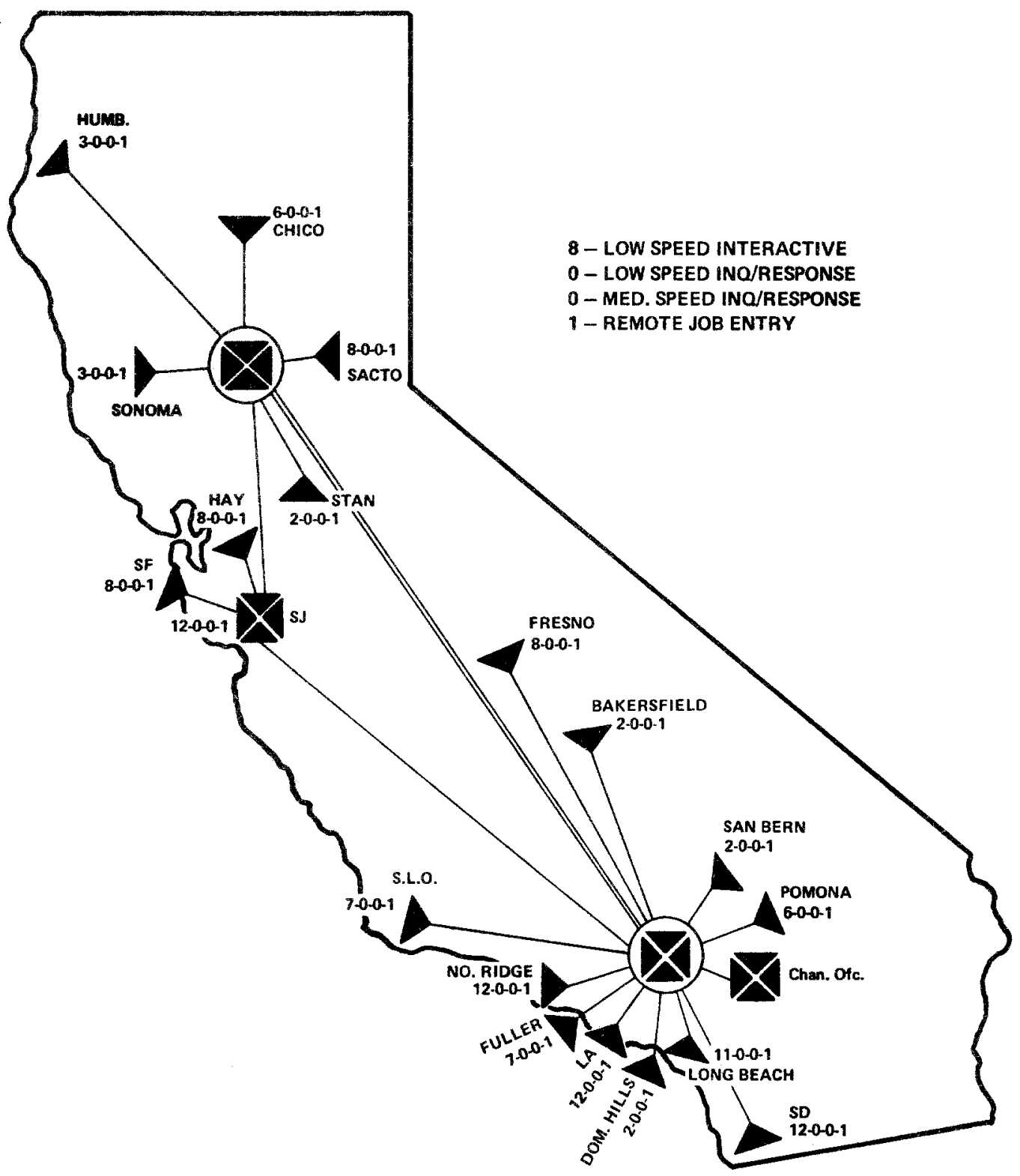

Figure 9 


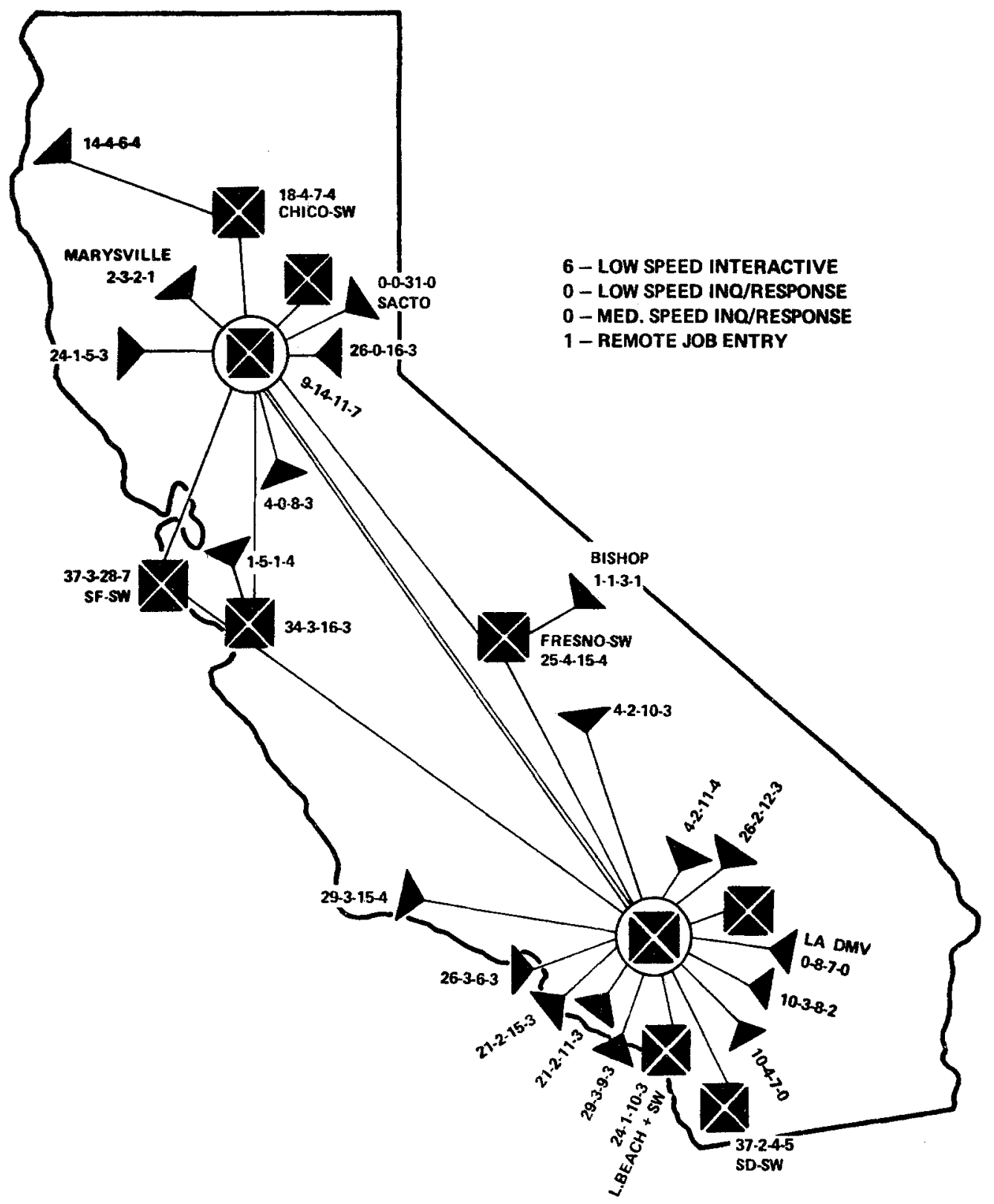

Figure 10 